JiFENG Chu (Nanjing)

DAQING JIANG (Changchun)

Donal O'Regan (Galway)

R. P. Agarwal (Melbourne, FL)

\title{
MULTIPLICITY OF POSITIVE SOLUTIONS TO SECOND ORDER DIFFERENTIAL EQUATIONS WITH NEUMANN BOUNDARY CONDITIONS
}

Abstract. We study the existence of positive solutions to second order nonlinear differential equations with Neumann boundary conditions. The proof relies on a fixed point theorem in cones, and the positivity of Green's function plays a crucial role in our study.

1. Introduction. In recent years many papers in the literature have discussed nonlinear differential equations with Neumann boundary conditions; see for example $[1-5,7,10,14,15]$. In this paper, we study the existence of positive solutions to the following Neumann boundary value problem:

$$
\left\{\begin{array}{l}
-x^{\prime \prime}+a(t) x=f(t, x), \quad 0 \leq t \leq 1, \\
x^{\prime}(0)=0, \quad x^{\prime}(1)=0,
\end{array}\right.
$$

where $a:[0,1] \rightarrow(0, \infty)$ is continuous and the nonlinearity $f:[0,1] \times(0, \infty)$ $\rightarrow(0, \infty)$ is continuous. If $a(t)=M>0$, we mention the following two results: In [9], Jiang and Liu obtained the existence of one positive solution of (1.1) when $f$ is either superlinear or sublinear, and in [14], Sun and Li gave some existence results for at least two positive solutions to (1.1) under weaker conditions than [9]. In the above two papers, existence results were obtained by using Krasnosel'skiil's fixed point theorem on compression and expansion of cones [8]. Another useful tool in establishing existence is the method of upper and lower solutions (see $[2,5,6,15])$.

2000 Mathematics Subject Classification: 34B15, 34B16.

Key words and phrases: positive solutions, Neumann boundary value problems, fixed point theorem in cones, Green's function.

Research of D. Q. Jiang supported by the NNSF of China. 
The aim of this paper is to study the existence of solutions of problem (1.1) by using a fixed point theorem in cones. To do so, we study the sign of the Green's function for the corresponding linear problem

$$
\left\{\begin{array}{l}
-x^{\prime \prime}+a(t) x=h(t), \quad 0 \leq t \leq 1 \\
x^{\prime}(0)=0, \quad x^{\prime}(1)=0
\end{array}\right.
$$

in Section 2. In particular, we construct the Green's function for problem (1.2) and prove its positivity. This fact is crucial in our arguments in Section 3. The Green's function for second order periodic boundary value problems was constructed and used in [11]. The sign property of the Green's function for differential equations with separated boundary conditions was investigated in $[12,13]$.

In Section 3, we will show, under simple and reasonable conditions, that (1.1) has at least one or two positive solutions by using a fixed point theorem in cones (see Theorem 2.5). Some examples are also given in Section 3.

2. Green's function and its positivity. In this section, we will consider the linear nonhomogeneous problem

$$
\left\{\begin{array}{l}
-x^{\prime \prime}+a(t) x=h(t), \quad 0 \leq t \leq 1 \\
x^{\prime}(0)=0, \quad x^{\prime}(1)=0 .
\end{array}\right.
$$

Lemma 2.1 ([11]). Let $K:[0,1] \times[0,1] \rightarrow[0, \infty)$ be a continuous function and $\psi$ be a nonnegative integrable function on $[0,1]$. Then for any nonnegative continuous function $\varphi$ defined on $[0,1]$, the Volterra integral equation

$$
x(t)=\varphi(t)+\int_{0}^{t} K(t, s) \psi(s) x(s) d s, \quad 0 \leq t \leq 1,
$$

has a unique solution $x(t)$. This solution is continuous and satisfies the inequality

$$
x(t) \geq \varphi(t), \quad 0 \leq t \leq 1 .
$$

Lemma 2.2. Let $u(t)$ and $v(t)$ be the solutions of the homogeneous equation

$$
-x^{\prime \prime}+a(t) x=0, \quad 0 \leq t \leq 1,
$$

satisfying the initial conditions $u(0)=1, u^{\prime}(0)=0, v(0)=0$ and $v^{\prime}(0)=1$. Then for any $s>t$, we have

$$
v^{\prime}(s)-t u^{\prime}(s) \geq 1 .
$$

Proof. Let $E(s, t)=v(s)-t u(s)$. To prove (2.4), we note that for fixed $t \in[0,1]$,

$$
\frac{\partial^{2} E(s, t)}{\partial s^{2}}=a(s) E(s, t)
$$


and

$$
E(0, t)=-t,\left.\quad \frac{\partial E(s, t)}{\partial s}\right|_{s=0}=1 .
$$

Hence it follows that, for all $s \in(t, 1]$, we have

$$
E(s, t)=(s-t)+\int_{0}^{s}(s-\xi) a(\xi) E(\xi, t) d \xi
$$

and

$$
\frac{\partial E(s, t)}{\partial s}=1+\int_{0}^{s} a(\xi) E(\xi, t) d \xi .
$$

By using Lemma 2.1, it follows from (2.5) and (2.6) that, for any $s>t$, we have

$$
E(s, t) \geq s-t>0, \quad \frac{\partial E(s, t)}{\partial s} \geq 1 .
$$

Therefore,

$$
v^{\prime}(s)-t u^{\prime}(s) \geq 1, \quad 0 \leq t<s \leq 1
$$

Theorem 2.3. Suppose $h:[0,1] \rightarrow[0, \infty)$ is continuous. Then problem (2.1) has a unique solution $x \in C^{2}([0,1])$ given by the formula

$$
x(t)=\int_{0}^{1} G(t, s) h(s) d s,
$$

where

$$
G(t, s)= \begin{cases}\frac{u(t) v^{\prime}(1)-v(t) u^{\prime}(1)}{u^{\prime}(1)} u(s), & 0 \leq s \leq t \leq 1, \\ \frac{u(s) v^{\prime}(1)-v(s) u^{\prime}(1)}{u^{\prime}(1)} u(t), & 0 \leq t \leq s \leq 1,\end{cases}
$$

is the Green's function and $u$ and $v$ are as in Lemma 2.2.

Proof. It is easy to see that the general solution to the equation

$$
-x^{\prime \prime}+a(t) x=h(t), \quad 0 \leq t \leq 1
$$

has the form

$$
x(t)=\alpha u(t)+\beta v(t)+\int_{0}^{t}[u(t) v(s)-u(s) v(t)] h(s) d s,
$$

where $\alpha$ and $\beta$ are arbitrary constants. Substituting this expression for $x(t)$ in the boundary conditions $x^{\prime}(0)=0, x^{\prime}(1)=0$, we obtain

$$
\beta=0 \quad \text { and } \quad \alpha=\frac{1}{u^{\prime}(1)} \int_{0}^{1}\left[u(s) v^{\prime}(1)-u^{\prime}(1) v(s)\right] h(s) d s .
$$

Now (2.7) and (2.8) are immediate. 
REMARK 2.4 ([9]). If $a(t)=m^{2}>0$, then the Green's function $G(t, s)$ of the boundary value problem (2.1) has the form

$$
G(t, s)= \begin{cases}\frac{\operatorname{ch} m(1-t) \operatorname{ch} m s}{m \operatorname{sh} m}, & 0 \leq s \leq t \leq 1, \\ \frac{\operatorname{ch} m(1-s) \operatorname{ch} m t}{m \operatorname{sh} m}, & 0 \leq t \leq s \leq 1,\end{cases}
$$

where

$$
\operatorname{ch} x=\frac{e^{x}+e^{-x}}{2}, \quad \operatorname{sh} x=\frac{e^{x}-e^{-x}}{2} .
$$

Theorem 2.5. Suppose that $a, h:[0,1] \rightarrow(0, \infty)$ are continuous functions. Then the Green's function $G(t, s)$ of problem $(2.1)$ is positive, i.e., $G(t, s)>0, t, s \in[0,1]$.

Proof. Since $G(t, s)=G(s, t)$, it is enough to prove that $G(t, s)>0$ for $0 \leq s \leq t \leq 1$. Using the initial conditions, we can easily deduce from equation (2.3) that

$$
\begin{aligned}
u(t) & =1+\int_{0}^{t}(t-s) a(s) u(s) d s, & v(t) & =t+\int_{0}^{t}(t-s) a(s) v(s) d s, \\
u^{\prime}(t) & =\int_{0}^{t} a(s) u(s) d s, & v^{\prime}(t) & =1+\int_{0}^{t} a(s) v(s) d s .
\end{aligned}
$$

It follows from Lemma 2.1 that

$$
u(t) \geq 1, \quad u^{\prime}(1)=\int_{0}^{1} a(s) u(s) d s \geq \int_{0}^{1} a(s) d s>0 .
$$

Now we prove

$$
F(t)=u(t) v^{\prime}(1)-v(t) u^{\prime}(1)>0, \quad \forall t \in[0,1] .
$$

Obviously,

$$
F^{\prime \prime}(t)=a(t) F(t), \quad 0 \leq t \leq 1 ; \quad F(0)=v^{\prime}(1), \quad F^{\prime}(0)=-u^{\prime}(1) .
$$

Hence, it follows that, for all $0 \leq t \leq 1$,

$$
F(t)=v^{\prime}(1)-t u^{\prime}(1)+\int_{0}^{t}(t-s) a(s) F(s) d s .
$$

It follows from Lemma 2.2 that $v^{\prime}(1)-t u^{\prime}(1) \geq 1$, so we have $F(t) \geq 1$ by applying Lemma 2.1.

In the arguments of Section 3 we need the following fixed point theorem in cones [8]. 
Theorem 2.6. Let $X$ be a Banach space and $K$ a cone in $X$. Assume $\Omega_{1}, \Omega_{2}$ are open subsets of $X$ with $0 \in \Omega_{1}, \bar{\Omega}_{1} \subset \Omega_{2}$. Let

$$
\Phi: K \cap\left(\bar{\Omega}_{2} \backslash \Omega_{1}\right) \rightarrow K
$$

be a continuous and completely continuous operator such that

(i) $\|\Phi x\| \leq\|x\|$ for $x \in K \cap \partial \Omega_{1}$;

(ii) there exists $\psi \in K \backslash\{0\}$ such that $x \neq \Phi x+\lambda \psi$ for $x \in K \cap \partial \Omega_{2}$ and $\lambda>0$.

Then $\Phi$ has a fixed point in $K \cap\left(\bar{\Omega}_{2} \backslash \Omega_{1}\right)$.

REMARK 2.7. In Theorem 2.6, if (i) and (ii) are replaced by

(i) ${ }^{*}\|\Phi x\| \leq\|x\|$ for $x \in K \cap \partial \Omega_{2}$;

(ii) $^{*}$ there exists $\psi \in K \backslash\{0\}$ such that $x \neq \Phi x+\lambda \psi$ for $x \in K \cap \partial \Omega_{1}$ and $\lambda>0$,

then $\Phi$ has also a fixed point in $K \cap\left(\bar{\Omega}_{2} \backslash \Omega_{1}\right)$.

Let

$$
A=\min _{0 \leq s, t \leq 1} G(t, s), \quad B=\max _{0 \leq s, t \leq 1} G(t, s), \quad \sigma=A / B .
$$

Thus $B>A>0$ and $0<\sigma<1$. In the applications below, we take $X=C([0,1])$ with the supremum norm $\|\cdot\|$ and define

$$
K=\left\{x \in X: x(t) \geq 0 \text { for all } t \text { and } \min _{0 \leq t \leq 1} x(t) \geq \sigma\|x\|\right\} .
$$

One may readily verify that $K$ is a cone in $X$. Suppose that $f:[0,1] \times(0, \infty)$ $\rightarrow(0, \infty)$ is continuous and we define an operator $\Phi: X \rightarrow X$ by

$$
(\Phi x)(t)=\int_{0}^{1} G(t, s) f(s, x(s)) d s
$$

for $x \in X$ and $t \in[0,1]$. It is easy to prove:

Lemma 2.8. $\Phi$ is well defined and maps $X$ into $K$. Moreover, $\Phi$ is continuous and completely continuous.

3. Multiplicity of positive solutions. In this section, we establish the existence and multiplicity of positive solutions to problem (1.1).

Theorem 3.1. Suppose that there exist $0<r<R$ such that $f(t, x) \geq 0$ for all $x \in[\sigma r, R]$. Then problem (1.1) has at least one positive solution if one of the following two conditions holds: 
(I) $f(t, x) \geq a(t) x, \quad \forall x \in[\sigma r, r], \quad f(t, x) \leq a(t) x, \quad \forall x \in[\sigma R, R]$;

(II) $f(t, x) \leq a(t) x, \quad \forall x \in[\sigma r, r], \quad f(t, x) \geq a(t) x, \quad \forall x \in[\sigma R, R]$.

Proof. The existence is proved using Theorem 2.6 and Remark 2.7. Define the open sets

$$
\Omega_{r}=\{x \in C([0,1]):\|x\|<r\}, \quad \Omega_{R}=\{x \in C([0,1]):\|x\|<R\} .
$$

Let $K$ be the cone defined by (2.11) and define an operator $\Phi$ on $K$ by (2.12). Clearly, $\Phi: K \cap\left(\bar{\Omega}_{R} \backslash \Omega_{r}\right) \rightarrow C([0,1])$ is continuous and compact since $f:[0,1] \times[\sigma r, R] \rightarrow \mathbb{R}$ is continuous. Also it is easy to see that $\Phi(K) \subset K$.

First, suppose that condition (I) holds.

Let $\psi \equiv 1$, so $\psi \in K$. Now we prove that

$$
x \neq \Phi x+\lambda \psi, \quad \forall x \in K \cap \partial \Omega_{r} \text { and } \lambda>0 .
$$

If not, there would exist $x_{0} \in K \cap \partial \Omega_{r}$ and $\lambda_{0}>0$ such that $x_{0}=\Phi x_{0}+\lambda_{0} \psi$. Since $x_{0} \in K \cap \partial \Omega_{r}$, it follows that $x_{0}(t) \geq \sigma\left\|x_{0}\right\|=\sigma r$. Let $\mu=\min _{t} x_{0}(t)$, so we have

$$
\begin{aligned}
x_{0}(t) & =\left(\Phi x_{0}\right)(t)+\lambda_{0}=\int_{0}^{1} G(t, s) f\left(s, x_{0}(s)\right) d s+\lambda_{0} \\
& \geq \int_{0}^{1} G(t, s) a(s) x_{0}(s) d s+\lambda_{0} \\
& \geq \mu \int_{0}^{1} G(t, s) a(s) d s+\lambda_{0}=\mu+\lambda_{0} ;
\end{aligned}
$$

note $\int_{0}^{1} G(t, s) a(s) d s=1$ for each $t$ (see Theorem 2.3 with $h=a$ ). This implies $\mu \geq \mu+\lambda_{0}$, a contradiction. Therefore, (3.1) holds.

Next we prove that

$$
\|\Phi x\| \leq\|x\|, \quad \forall x \in K \cap \partial \Omega_{R} .
$$

In fact, for any $x \in K \cap \partial \Omega_{R}$, we have

$$
\begin{aligned}
(\Phi x)(t) & =\int_{0}^{1} G(t, s) f(s, x(s)) d s \leq \int_{0}^{1} G(t, s) a(s) x(s) d s \\
& \leq \int_{0}^{1} G(t, s) a(s) d s \cdot \max _{t} x(t)=\|x\| .
\end{aligned}
$$

Therefore, $\|\Phi x\| \leq\|x\|$, i.e., (3.2) holds.

It follows from Remark 2.7, (3.1) and (3.2) that $\Phi$ has a fixed point $x \in K \cap\left(\bar{\Omega}_{R} \backslash \Omega_{r}\right)$. Clearly, this fixed point is a positive solution of (1.1) satisfying $r \leq\|x\| \leq R$. 
If condition (II) holds, then similar reasoning yields

$$
\|\Phi x\| \leq\|x\|, \quad \forall x \in K \cap \partial \Omega_{r},
$$

and

$$
x \neq \Phi x+\lambda \psi, \quad \forall x \in K \cap \partial \Omega_{R} \text { and } \lambda>0 .
$$

Then it follows from Theorem 2.6, (3.3) and (3.4) that $\Phi$ has also a fixed point $x \in K \cap\left(\bar{\Omega}_{R} \backslash \Omega_{r}\right)$.

Corollary 3.2. Assume that $f(t, x) \geq 0$ for all $(x, t) \in(0, \infty) \times[0,1]$. Then problem (1.1) has at least one positive solution if one of the following two conditions holds:

(I) (Superlinear)

$$
\lim _{x \rightarrow 0^{+}} \frac{f(t, x)}{x}=0, \quad \lim _{x \rightarrow \infty} \frac{f(t, x)}{x}=\infty, \quad \text { uniformly for } t \in[0,1] ;
$$

(II) (Sublinear)

$$
\lim _{x \rightarrow 0^{+}} \frac{f(t, x)}{x}=\infty, \quad \lim _{x \rightarrow \infty} \frac{f(t, x)}{x}=0, \quad \text { uniformly for } t \in[0,1] .
$$

Proof. This follows immediately from Theorem 3.1 by taking $r$ small enough and $R$ large enough.

TheOREm 3.3. Suppose that there exist $0<r<p<R$ such that

$$
f(t, x) \geq 0, \quad \forall x \in[\sigma r, R] .
$$

Then problem (1.1) has at least two positive solutions if one of the following two conditions holds:

(I) $f(t, x) \geq a(t) x, \forall x \in[\sigma r, r] ; f(t, x)<a(t) x, \forall x \in[\sigma p, p] ; f(t, x) \geq$ $a(t) x, \forall x \in[\sigma R, R]$

(II) $f(t, x) \leq a(t) x, \forall x \in[\sigma r, r] ; f(t, x)>a(t) x, \forall x \in[\sigma p, p] ; f(t, x) \leq$ $a(t) x, \forall x \in[\sigma R, R]$.

Proof. We only prove the result when condition (I) holds since similar reasoning establishes the result for condition (II).

Define $\Omega_{r}, \Omega_{R}, K$ and $\Phi$ as in Theorem 3.1 and define $\Omega_{p}=$ $\{x \in C([0,1]):\|x\|<p\}$. As in the proof of Theorem 3.1, it is easy to see that

$$
\begin{gathered}
x \neq \Phi x+\lambda \psi, \quad \forall x \in K \cap \partial \Omega_{r} \text { and } \lambda>0 ; \\
x \neq \Phi x+\lambda \psi, \quad \forall x \in K \cap \partial \Omega_{R} \text { and } \lambda>0 ; \\
\|\Phi x\|<\|x\| \quad \forall x \in K \cap \partial \Omega_{p} .
\end{gathered}
$$

Now Theorem 2.6 (applied with $\Omega_{1}=\Omega_{p}$ and $\Omega_{2}=\Omega_{R}$ ) guarantees that there exists a solution $x_{2}$ with $p \leq\left\|x_{2}\right\| \leq R$. Note $\left\|x_{2}\right\|>p$ 
from (3.7). Also Remark 2.7 (applied with $\Omega_{2}=\Omega_{p}$ and $\Omega_{1}=\Omega_{r}$ ) guarantees that there exists a solution $x_{1}$ with $r \leq\left\|x_{1}\right\| \leq p$. Note $\left\|x_{1}\right\|<p$ from (3.7).

In fact, we can derive some results from Theorem 3.3 if we assume appropriate asymptotic behavior of the nonlinearity. We introduce the following hypotheses:

$\left(\mathrm{H}_{1}\right) \lim _{x \rightarrow 0^{+}} \frac{f(t, x)}{x}=\infty, \quad \lim _{x \rightarrow \infty} \frac{f(t, x)}{x}=\infty, \quad$ uniformly in $t \in[0,1]$.

$\left(\mathrm{H}_{2}\right) \lim _{x \rightarrow 0^{+}} \frac{f(t, x)}{x}=0, \quad \lim _{x \rightarrow \infty} \frac{f(t, x)}{x}=0, \quad$ uniformly in $t \in[0,1]$.

$\left(\mathrm{H}_{3}\right)$ There exists a $p>0$ such that $\sigma p \leq x \leq p$ implies $f(t, x)<a(t) p$, $t \in[0,1]$.

$\left(\mathrm{H}_{4}\right)$ There exists a $p>0$ such that $\sigma p \leq x \leq p$ implies $f(t, x)>a(t) x$, $t \in[0,1]$.

Corollary 3.4. Assume that $f(t, x) \geq 0$ for all $(x, t) \in(0, \infty) \times[0,1]$. Then problem (1.1) has at least two positive solutions satisfying $0<\left\|x_{1}\right\|<$ $p<\left\|x_{2}\right\|$ provided that conditions $\left(\mathrm{H}_{1}\right)$ and $\left(\mathrm{H}_{3}\right)$ (respectively $\left(\mathrm{H}_{2}\right)$ and $\left.\left(\mathrm{H}_{4}\right)\right)$ hold.

Proof. This follows from Theorem 3.3 by taking $r$ small enough and $R$ large enough.

EXAMPLE 3.5. Suppose the nonlinearity in problem (1.1) is

$$
f(t, x)=\mu b(t)\left(x^{\alpha}+x^{\beta}\right),
$$

where $0<\alpha<1, \beta>0, b \in C([0,1])$ is a positive function, and $\mu>0$ is a positive parameter.

(i) If $\beta<1$, (1.1) has at least one positive solution for each $\mu>0$.

(ii) If $\beta \geq 1$, (1.1) has at least two positive solutions for each $0<\mu<\mu_{*}$, where $\mu_{*}$ is some positive constant.

Proof. First we discuss (i). It is easy to see that

(3.9) $\lim _{x \rightarrow 0^{+}} \frac{f(t, x)}{x}=\infty, \quad \lim _{x \rightarrow \infty} \frac{f(t, x)}{x}=0, \quad$ uniformly in $t \in[0,1]$,

if $0<\alpha, \beta<1$. The result now follows from Corollary 3.2(II).

Next we discuss (ii). Notice that

$$
\lim _{x \rightarrow 0^{+}} \frac{f(t, x)}{x}=\infty, \quad \lim _{x \rightarrow \infty} \frac{f(t, x)}{x}=\infty, \quad \text { uniformly in } t \in[0,1],
$$

since $0<\alpha<1$ and $\beta>1$. Set

$$
T(x)=\frac{x}{x^{\alpha}+x^{\beta}}, \quad x>0 .
$$


Then $T\left(0^{+}\right)=T(\infty)=0$ and

$$
T(x) \leq T(p)=\sup _{x \in(0, \infty)} T(x), \quad \text { where } p=\left(\frac{1-\alpha}{\beta-1}\right)^{1 /(\beta-\alpha)} .
$$

Let $\mu^{*}=\sigma T(p) e^{-1}$, where $e=\max _{t} b(t) / a(t)$. Then for $x \in[\sigma p, p]$ and $\mu \in\left(0, \mu_{*}\right)$, we have

$$
\begin{aligned}
f(t, x) & =\mu b(t)\left(x^{\alpha}+x^{\beta}\right)<\mu^{*} a(t)\left(p^{\alpha}+p^{\beta}\right) \max _{t} \frac{b(t)}{a(t)} \\
& =\sigma T(p) a(t)\left(p^{\alpha}+p^{\beta}\right)=\sigma a(t) p<a(t) p .
\end{aligned}
$$

Now (3.10) and (3.11) imply that conditions $\left(\mathrm{H}_{1}\right)$ and $\left(\mathrm{H}_{3}\right)$ are satisfied, so the existence of two solutions is guaranteed by applying Corollary 3.4.

Our main results can also be applied to the singular case.

EXAMPLE 3.6. Let the nonlinearity in (1.1) be

$$
f(t, x)=b(t) x^{-\alpha}+\mu c(t) x^{\beta}+e(t), \quad 0 \leq t \leq 1,
$$

where $\alpha>0, \beta \geq 0, b, c, e \in C([0,1])$ are nonnegative functions with $b(t)>0$ for all $t$, and $\mu>0$ is a positive parameter.

(i) If $\beta<1,(1.1)$ has at least one positive solution for each $\mu>0$.

(ii) If $\beta>1,(1.1)$ has at least two positive solutions for each $0<\mu<\mu_{*}$, where $\mu_{*}$ is some positive constant.

Proof. First we discuss (i). It is easy to see that

$$
\lim _{x \rightarrow 0^{+}} \frac{f(t, x)}{x}=\infty, \quad \lim _{x \rightarrow \infty} \frac{f(t, x)}{x}=0, \quad \text { uniformly in } t \in[0,1],
$$

if $\alpha>0,0<\beta<1$. The result follows from Corollary 3.2(II).

Next we prove (ii). Notice that

(3.14) $\lim _{x \rightarrow 0^{+}} \frac{f(t, x)}{x}=\infty, \quad \lim _{x \rightarrow \infty} \frac{f(t, x)}{x}=\infty, \quad$ uniformly in $t \in[0,1]$, since $0<\alpha<1$ and $\beta>1$. Set

$$
T(x)=\frac{a_{l} \sigma^{\alpha} x^{\alpha+1}-e_{0} \sigma^{\alpha} x^{\alpha}-b_{0}}{c_{0} \sigma^{\alpha} x^{\alpha+\beta}},
$$

where

$$
a_{l}=\min _{t} a(t), \quad b_{0}=\max _{t} b(t), \quad c_{0}=\max _{t} c(t), \quad e_{0}=\max _{t} e(t) .
$$

It is easy to see that there exists $x^{*} \in(0, \infty)$ such that $T\left(x^{*}\right)=0$ since $T\left(0^{+}\right)<0$. Therefore, there exists $p \in\left(x^{*}, \infty\right)$ such that

$$
T(x) \leq T(p)=\sup _{x \in\left(x^{*}, \infty\right)} T(x)
$$


since $T\left(x^{*}\right)=T(\infty)=0$. Let

$$
\mu_{*}=T(p)=\frac{a_{l} \sigma^{\alpha} p^{\alpha+1}-e_{0} \sigma^{\alpha} p^{\alpha}-b_{0}}{c_{0} \sigma^{\alpha} p^{\alpha+\beta}} .
$$

Then for $x \in[\sigma p, p]$ and $\mu \in\left(0, \mu_{*}\right)$, we have

$$
\begin{aligned}
f(t, x) & =b(t) x^{-\alpha}+\mu c(t) x^{\beta}+e(t)<b_{0}(\sigma p)^{-\alpha}+\mu_{*} c_{0} p^{\beta}+e_{0} \\
& =\frac{b_{0}}{\sigma^{\alpha} p^{\alpha}}+\frac{a_{l} \sigma^{\alpha} p^{\alpha+1}-e_{0} \sigma^{\alpha} p^{\alpha}-b_{0}}{\sigma^{\alpha} p^{\alpha}}+e_{0} \\
& =a_{l} p<a(t) p .
\end{aligned}
$$

Now (3.14) and (3.15) imply that conditions $\left(\mathrm{H}_{1}\right)$ and $\left(\mathrm{H}_{3}\right)$ of Corollary 3.4 are satisfied, so the existence of two solutions is guaranteed.

\section{References}

[1] A. Cabada and P. Habets, Optimal existence conditions for $\phi$-Laplacian equations with upper and lower solutions in the reversed order, J. Differential Equations 166 (2000), 385-401.

[2] A. Cabada, P. Habets and S. Lois, Monotone method for the Neumann problem with lower and upper solutions in the reverse order, Appl. Math. Comput. 117 (2001), $1-14$.

[3] A. Cabada and R. R. L. Pouse, Existence result for the problem $\left(\phi\left(u^{\prime}\right)\right)^{\prime}=f\left(t, u, u^{\prime}\right)$ with periodic and Neumann boundary conditions, Nonlinear Anal. 30 (1997), $1733-1742$.

[4] A. Cabada and L. Sanchez, A positive operator approach to the Neumann problem for a second order ordinary differential equation, J. Math. Anal. Appl. 204 (1996), $774-785$.

[5] M. Cherpion, C. De Coster and P. Habets, A constructive monotone iterative method for second order BVP in the presence of lower and upper solutions, Appl. Math. Comput. 123 (2001), 75-91.

[6] C. De Coster and P. Habets, Upper and lower solutions in the theory of ODE boundary value problems: Classical and recent results, in: Nonlinear Analysis and Boundary Value Problems for Ordinary Differential Equations, F. Zanolin (ed.), CISM-ICMS 371, Springer, New York, 1996, 1-78.

[7] H. Dang and S. F. Oppenheimer, Existence and uniqueness results for some nonlinear boundary value problems, J. Math. Anal. Appl. 198 (1996), 35-48.

[8] K. Deimling, Nonlinear Functional Analysis, Springer, New York, 1985.

[9] D. Q. Jiang and H. Z. Liu, Existence of positive solutions to second order Neumann boundary value problem, J. Math. Res. Exposition 20 (2000), 360-364.

[10] R Y. Ma, Existence of positive radial solutions for elliptic systems, J. Math. Anal. Appl. 201 (1996), 375-386.

[11] F. Merivenci Atici and G. Sh. Guseinov, On the existence of positive solutions for nonlinear differential equations with periodic boundary conditions, J. Comput. Appl. Math. 132 (2001), 341-356.

[12] A. C. Peterson, On the sign of Green's function beyond the interval of disconjugacy, Rocky Mountain J. Math. 3 (1973), 41-51. 
[13] A. C. Peterson, On the sign of Green's functions, J. Differential Equations 21 (1976), 167-178.

[14] J. P. Sun and W. T. Li, Multiple positive solutions to second order Neumann boundary value problems, Appl. Math. Comput. 146 (2003), 187-194.

[15] N. Yazidi, Monotone method for singular Neumann problem, Nonlinear Anal. 49 (2002), 589-602.

Department of Mathematics and Physics

Hohai University

Nanjing 210098, China

Department of Mathematics

National University of Ireland

Galway, Ireland

E-mail: donal.oregan@nuigalway.ie
Department of Mathematics Northeast Normal University Changchun 130024, Jilin, China E-mail: daqingjiang@vip.163.com

Department of Mathematical Science Florida Institute of Technology Melbourne, FL 32901-6975, U.S.A. E-mail: agarwal@fit.edu

Received on 27.8.2004;

revised version on 21.4.2005 